\title{
Modelado de riesgos para el sistema vaca-cría del estado de Chihuahua utilizando indicadores socioeconómicos y ambientales
}

\section{Modeling of risk in the cow-calf system of the state of Chihuahua with socio-economic and environmental indicators}

\author{
I reyli Zuluamy Iracheta-Laraa , Eva I glesias-Martínezb, Gerardo Méndez-Zamorac, Mario Edgar Esparza-Velaa , \\ Eduardo Santellano-Estrada ${ }^{a^{*}}$
}

\section{RESUMEN}

Los sistemas de producción de bovinos-carne se encuentran expuestos a una vulnerabilidad inminente por el sobre pastoreo, cambios en el uso de suelo y un manejo inadecuado del hato. El objetivo de esta investigación fue elaborar un modelo matemático integrado por indicadores ambientales y socioeconómicos de tres ecorregiones, que identifiquen factores y patrones de vulnerabilidad en la densidad ganadera ( $\mathrm{Dg}$ ) del sistema vaca-cría del estado de Chihuahua. La estimación mostró que el aumento de la Dg en una unidad animal en los predios ejidales eleva los costos de producción 0.0358 unidades porcentuales, al demandar un mayor consumo de materia vegetal e insumos. La diferencia en las unidades animal entre tipos de tenencia predial, al considerar un programa de capacitación técnico pecuario, disminuye entre las zonas, y mostró que la vulnerabilidad en los predios ejidales es inferior ante un evento de sequía. Se pretende que este trabajo genere información útil para analizar los posibles riesgos que afecten la rentabilidad económica, social y ambiental en las ecorregiones del estado de Chihuahua.

PALABRAS CLAVE: Riesgo, Ecorregiones, Sensores remotos, Sistema de producción.

\begin{abstract}
Overgrazing, changes in land use and inadequate management that supports it exposes the cattle-beef systems to an imminent vulnerability. The aim of this research was to develop an integrated model of environmental and socioeconomic indicators of three ecoregions, identifying vulnerability factors and patterns of cow-calf system in the grasslands of the State of Chihuahua that affects the livestock density (LD). The estimation showed that the increasing of LD in the Ejido lands raises the production costs 0.0358 units, by demanding a higher materials and forage intake. The difference in animal units between farm types (considering a technical program of training livestock) decreases depending the areas, and shows that the Ejidos lands are less vulnerable in time of drought. This report will generate useful information for analyzing the potential economic, social and environmental risks in the ecoregions of the state of Chihuahua.
\end{abstract}

KEY WORDS: Risk, Ecoregions, Remote sensing, Production system.

\footnotetext{
Recibido el 8 de abril de 2015. Aceptado el 1 de agosto de 2015

${ }^{a}$ Facultad de Zootecnia y Ecología, Universidad Autónoma de Chihuahua. Periférico R. Almada km. 1, 31453, Chihuahua, México.

${ }^{b}$ Centro de Estudios e Investigación para la Gestión de Riesgos Agrarios y Medioambientales, Universidad Politécnica de Madrid. España.

' Facultad de Agronomía, Universidad Autónoma de Nuevo León. Escobedo, N.L. México.

* Autor de correspondencia: esantellanoe@gmail.com.
} 
El sistema vaca-cría es una actividad económica tradicional en el estado de Chihuahua; sin embargo, en la última década su rentabilidad ha decrecido debido a la baja productividad de los pastizales. Al respecto, en Chihuahua ha sido documentado el impacto de la precipitación y temperatura en la productividad de pastizales desérticos y semidesérticos ${ }^{(1,2,3)}$. Por ejemplo, estudios han reportado que en el periodo 2009-2011 hubo sequía extrema, la segunda más intensa en 60 años ${ }^{(4-7)}$, donde la precipitación (58 $\mathrm{mm}$ ) en 2011 fue la más baja en registros históricos ${ }^{(8)}$. Así mismo, el Monitor de Sequía de América del Norte $^{(9)}$ documentó que el $85 \%$ de los municipios de Chihuahua estuvieron en sequía extrema hasta el mes de abril del año 2012. En promedio, las precipitaciones han disminuido $60 \%$ en la última década para las tres ecorregiones de Chihuahua, reduciendo la producción de pastizales, la captación de agua para abrevadero y la productividad ganadera (menor porcentaje de parición y alta mortalidad) ${ }^{(10)}$. Por destacar, posterior a la sequía en 2011 se afectaron más de 2.5 millones de ganaderos con pérdidas superiores a los 500,000 millones de dólares(11).

En relación, la variabilidad climática, los cambios de uso del suelo y el sobrepastoreo provocan que la disponibilidad de forraje sea crítica en las ecorregiones ganaderas desérticas de Chihuahua(12). Esta problemática ha suscitado una baja rentabilidad del hato y un retroceso en el capital financiero de grandes y pequeños productores, reflejándose en altos costos de producción y baja exportación de becerros, por lo que es necesario una modelación que pronostique los principales riesgos económicos y el comportamiento del hato. De no tomar medidas oportunas, en el estado podrían afectarse 36,758 núcleos agrarios, 25,346 ejidos y 11,412 predios particulares, los cuales tienen más del $28 \%$ de la superficie total del Estado(13). De las 24.7 millones hectáreas de Chihuahua, 18 millones son de agostadero, que contribuye con $6.36 \%$ al producto interno bruto (PIB) estatal y, junto con el estado de Durango, Guanajuato, Zacatecas y Veracruz, aportan el $44.7 \%$ al PIB nacional agropecuario, donde Chihuahua destaca por participar como el principal exportador de becerros del país(14).

Basado en lo anterior, es necesario diseñar un modelo que permita definir y cuantificar los riesgos en la ganadería estatal, incluyendo las sequias asociadas a los efectos del cambio climático. La utilización de herramientas y métodos geoespaciales, así como la integración de modelos econométricos permiten analizar variables relacionadas con las características ambientales y productivas de los pastizales, al comparar datos cualitativos y cuantitativos $^{(15,16)}$. Así, modelos biofísicos pueden usarse para analizar el crecimiento y disponibilidad de la vegetación y su interacción con datos climáticos y socioeconómicos ${ }^{(17,18)}$, así como para analizar el impacto de un cambio aleatorio en alguna de las variables del sistema vaca-cría(19) y proponer soluciones a los riesgos en la productividad ganadera en un futuro determinado.

No obstante, la determinación de riesgo mediante modelos y simulaciones, es una herramienta escasamente usada y poco adoptada en las investigaciones pecuarias para la región norte de México. Por ello, el objetivo de esta investigación fue elaborar un modelo matemático integrado por indicadores ambientales y socioeconómicos que evalúe el comportamiento de la densidad ganadera del sistema vaca-cría en los pastizales de tres ecorregiones del estado de Chihuahua.

\section{Área de estudio}

En el estado de Chihuahua se estudiaron tres regiones ecológicas: Desierto, Valles Centrales o Semiárida y Sierra Tarahumara. La región del desierto tiene un clima seco semicálido; las temperaturas exceden $40{ }^{\circ} \mathrm{C}$ durante el verano y -5 ${ }^{\circ} \mathrm{C}$ en invierno. En la zona serrana predomina un clima subhúmedo semifrío a subhúmedo templado, con nevadas invernales abundantes; esta zona montañosa sobrepasa los 2,400 msnm, con serranías alargadas y angostas de 200 a 1,000 m sobre la Ilanura. En las Ilanuras occidentales, las temperaturas mínimas son las más bajas del estado con $-20{ }^{\circ} \mathrm{C}$, la precipitación varía de 300 a $550 \mathrm{~mm}$ y predomina un clima subhúmedo templado a semidesértico templado(20).

\section{Obtención de los datos y variables}

La información base del análisis y la generación del modelo se conformó por un panel de 64,062 predios, distribuidos en los 67 municipios de 
Chihuahua, con datos anuales tomados en el periodo 2008-2013 de las tres ecorregiones. Así, variables económicas, sociales y ambientales se utilizaron para estudiar el comportamiento de la densidad ganadera.

\section{Variables ambientales}

Índice Diferenciado de Vegetación Normalizada (NDVl; valores medios de predios ejidales y privados de julio a octubre; como indicador el ciclo de recuperación natural de los pastizales en las zonas áridas y desérticas del estado $\left.{ }^{(2)}\right)$. Las imágenes se tomaron de LANDSAT 7 ETM y LANDSAT 8 (tamaño de imagen 30 × $30 \mathrm{~m}$ de resolución), aplicando corrección geométrica, radiométrica y atmosférica para disminuir el efecto de ruido ${ }^{(21)}$; precipitación y temperatura (Precip y Temp; representados por la cantidad de milímetros cúbicos acumulados anualmente por municipio, así como la temperatura media mensual); y tamaño de la superficie (número de hectáreas del predio).

\section{Variables económicas}

Densidad ganadera (Dg; unidades animal (UA)/ha predio). Capacidad de carga ${ }^{(14)}$ basados en la información estatal del Registro Agrario Nacional y paneles regionales de productores (esta variable puede estar subestimada porque algunos productores no reportaron sus unidades animal, debido a la inseguridad en el Estado en 2008); comportamiento económico en los predios (Prod; promedio de la producción pecuaria, comparada con la densidad ganadera existente, periodo 2008-2013; ingresos netos por exportaciones); costos pecuarios (Cost; egresos promedio por municipio, fijos y variables, de las explotaciones).

\section{Variables sociales}

Variable Ejido RAN (22) (representación dicotómica de los tipos de tenencia con mayor número de explotaciones en Chihuahua). Esta variable se clasificó en predios privados y ejidales para comparar la producción ganadera de los productores pecuarios en el Estado, tomando valor 1 para los predios identificados como ejidos y 0 para los privados; número de productores pecuarios capacitados en predios privados y ejidales en 2010-
2012 también fue incluido (programa de Desarrollo de Capacidades, Innovación Tecnológica y Extensionismo Rural establecido por SAGARPA $\left.{ }^{(23)}\right)$. Para identificar la tendencia temporal del modelo se generó una variable binaria (Post2011; variable dicotómica del cambio estructural posterior al año de impacto $2011^{(9)}$; donde 1 se asignó a todos los años posteriores al 2010 y 0 a los anteriores).

\section{Especificación del modelo}

Considerando las variables Dg y NDVI, la variable binomial Post2011 se creó mediante el Test Chow; ésta es la desviación entre el periodo final e inicial (al considerar el año 2011 como línea base; Post2011=1). Así, el coeficiente de Dg se definió como la pendiente de la línea base del periodo y, el coeficiente de Dg_post2011 fue la desviación de la pendiente entre ambos grupos. De esta manera, la hipótesis nula fue que los periodos tienen iguales parámetros para la densidad ganadera y para el intercepto. El modelado de esta investigación se llevó a cabo en tres etapas, debido a la falta de ajuste para el norte de México de los modelos integrados (variables ambientales e imágenes satelitales), y la poca disponibilidad de factores socioeconómicos. El primer modelo definido fue:

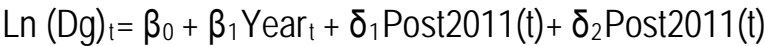

${ }^{*}{ }^{*} \mathrm{Dear}_{\mathrm{t}}+\xi$

Donde $\mathrm{Ln}=$ indica presencia de logaritmo natural en los parámetros $\beta_{0}$ y $\beta_{1} ;(D g)_{t}=$ variable dependiente (unidades animal/ha/año); Year $_{\mathrm{t}}=$ variable entera que capta la tendencia; Post $2011_{\mathrm{t}}=$ variable de cambio, toma valor cero $(t=0)$ para los años que transcurren antes de la sequía y valor uno $(t=1)$ para los años posteriores al 2011; $t=$ marca el año seleccionado; $\beta_{0}=$ refleja el cambio en el coeficiente de la "pendiente"; $\beta_{1}=$ alteración en la media de la variable endógena; $\delta_{1}=$ parámetro de agrupación del primer periodo; $\delta_{2}=$ parámetro de agrupación del segundo periodo; $\xi=$ término de error. La hipótesis de que existió un cambio en la media como resultado en la tendencia de la serie temporal es descrita por medio de la prueba de $\mathrm{F}$, la cual tiene como hipótesis nula que $\delta_{1}-\delta_{2}=0$.

Para la segunda etapa y después de la estimación del cambio estructural en la serie de 
datos donde todos los parámetros fueron establecidos e integrados, se obtuvo el siguiente modelo:

$\operatorname{Ln}(\mathrm{Dg})_{\mathrm{it}}=\beta_{0}+\beta_{1} \mathrm{LnNDV}_{\mathrm{it}}+\beta_{2} \mathrm{LnPrecipit}_{\mathrm{it}}+\beta_{3}$ LnTemp $_{\text {it }}+$ $\beta_{4}$ LnSize $_{i t}+\beta_{5}$ Ejido $_{i t}+\beta_{6}$ LnCost $_{i t}+\beta_{7}$ LnProd $_{i t}+\beta_{8}{\text { post } 2011_{i t}}+\xi_{\text {it }}$

Siendo: $\operatorname{Ln}(\mathrm{Dg})_{\mathrm{it}}=$ logaritmo natural de la variable dependiente (unidades animal/ha/año/ predio); $\beta_{0}=$ la ordenada al origen; $\beta_{1} \mathrm{LnNDVI}_{\text {it }}=$ disponibilidad de materia vegetal (año/predio); $\beta_{2}$ LnPrecipit $_{i t}=$ precipitación media; $\beta_{3}$ LnTemp $_{\mathrm{it}}=$ temperatura media anual; $\beta_{4}$ LnSize $_{i t}=$ superficie de los predios (ha/predio); $\beta_{5}$ Ejido $_{\text {it }}=$ variable descriptiva, toma 0 (ejido $=0$ ) para los predios privados y 1 para los predios comunales (ejido $=1$ ); $\beta_{6}$ LnCost $_{\text {it }}=$ egresos netos de las explotaciones

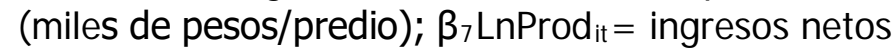
por unidad animal (miles de pesos/UA); $\beta_{8}$ post $2011_{\text {it }}=$ variable de cambio, toma valor cero $(\mathrm{t}=0)$ para los años que transcurren antes de la sequía y valor uno $(t=1)$ para los años posteriores al 2011.

El modelo especificado presentó algunos desafíos para su estimación, ya que algunas de las variables explicativas son endógenas. En particular, el NDVI depende de la precipitación y temperatura de la zona; por lo tanto, potencialmente correlacionado con los términos de error del índice.

Interacciones entre las variables generales y las dicotómicas de tiempo (post2011) y de tipo (ejido) fueron integradas, y el comportamiento general de las explotaciones se explicó. Así, tres variables binarias (dicotómicas) se establecieron en las zonas para identificar el comportamiento no observado de la variable dependiente $(\mathrm{Dg})$; entonces, $\mathrm{k}-1$ zonas descritas en el modelo se obtuvieron y, el valor de $P$ de la prueba de $\mathrm{F}$ para conocer la significancia conjunta de las variables dicotómicas en el modelo $\left(\mathrm{n}_{1}=\mathrm{n}_{2}=\ldots=\mathrm{n}_{\mathrm{k}}=0\right)$ no se pudo rechazar, por lo tanto se afirma que las variables dicotómicas son significativas. Finalmente en la tercera etapa, el modelo estimado fue:

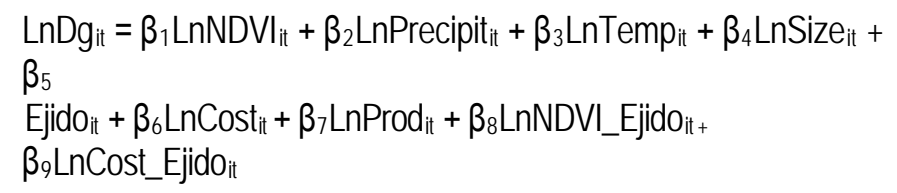

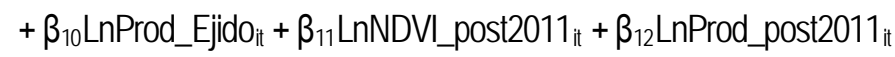
$+\beta_{13}$ LnCost_post2011 it $+\beta_{14}$ LnBenef-2010 it $+\beta_{15}$ LnBenef-2011 $+\beta_{16}$ LnBenef-2012 it $+\beta_{17}$ post2011 $1_{\text {it }}+\delta_{2} z_{2}+\delta_{3} Z_{3}+\xi_{\text {it. }}$.

Además de los parámetros de la ecuación (2), las siguientes variables se incorporaron: $\beta_{8}$ LnNDVI Ejido $_{\text {it }}=$ relación de la disponibilidad vegetal y el tipo de tenencia del predio (kg/ha/predio); ßgLnCost Ejido $_{\text {it }}=$ relaciona el egreso neto de los predios definidos con tenencia ejidal; $\beta_{10} L_{\text {nProd_Ejido }}=\mathrm{el}$ ingreso neto por unidad animal en los predios con tenencia ejidal; $\quad \beta_{11}$ LnNDVI_post $2011_{i t}=$ la producción de materia vegetal posterior al año 2011; $\beta_{12}$ LnProd_post $2011_{\text {it }}=$ ingreso neto económico por unidad animal posterior al año 2011; $\beta_{13}$ LnCost_post $2011_{i t}=$ el egreso neto por unidad animal posterior al año $2011 ; \beta_{14}$ LnBenef-2010it $=$ interacción de los beneficiarios para el año 2010; $\beta_{15}$ LnBenef- $2011_{i t}=$ número de beneficiarios del programa para el año 2011; $\beta_{16}$ LnBenef-2012it $=$ iteración de los beneficiarios para el año 2012; $\beta_{17}$ post $2011_{\mathrm{it}}=$ variable binaria que agrupa el periodo posterior al año de sequía (2011).

En cuanto al signo y valor esperado de los parámetros de la ecuación (3), se espera que la Dg sea menor en los predios catalogados como ejidales $^{(24)}\left(\beta_{5}<0\right) \quad y$, que la variación de la precipitación provoque que la densidad animal sea menor cuando la precipitación disminuya $\left(\beta_{2}<0\right)$, y a medida que la disponibilidad de materia vegetal aumente, se incremente la $\mathrm{Dg}\left(\beta_{1}>0\right)$.

También, para observar si existió una mejora en la planificación y utilización de los predios, posterior a la implementación del programa de capacitación, se estableció una relación negativa cuando el ingreso de la producción $\left(\beta_{7}<0\right)$ y los costos de la actividad ganadera $\left(\beta_{6}<0\right)$ aumentaron.

\section{Análisis del modelado}

Para elaborar el modelo econométrico y desarrollar los patrones de conducta de las variables se utilizó el software STATA ${ }^{\circledR(25)}$. La definición del modelo regresivo involucró el análisis de efectos fijos y aleatorios para controlar la heterogeneidad de los datos de un panel; esto a través del modelo general de regresión lineal (comando regress), modelo con un efecto aleatorio y fijo (comando xtreg), modelo de 
efectos fijos autoregresivo de grado 1 (AR1; comando xtregar) y los modelos para controlar problemas de heterogeneidad, autocorrelación, heterocedasticidad y correlación contemporánea (comando xtgls y xtpcse). El tratamiento a las imágenes satelitales y los análisis radiales para las variables de precipitación y temperatura se realizó con ArcGIS 9.3 ${ }^{\circledR(26)}$.

Un cambio estructural se reflejó para la serie de datos en el comportamiento de la densidad ganadera (Dg) municipal de Chihuahua en el segundo periodo (2011-2013); resultado obtenido del análisis de la Dg en los periodos 2008-2010 y 2011-2013 (se rechazó la hipótesis nula $\delta_{1}=\delta_{2}$; ecuación 1). Para la especificación del modelo en la segunda fase de análisis (ecuación 2), los efectos aleatorios $u_{i}$ fueron relevantes (prueba Breusch Pagan y Hausman), por lo que resultó pertinente estimarlos. Por lo tanto, la diferencia entre los coeficientes de efectos aleatorios y fijos del modelo no fue sistemática, lo que sugirió utilizar el modelo con efectos aleatorios.

Finalmente, en la tercera fase de la estimación y análisis del tercer modelo (ecuación 3) derivado del análisis de las variables estudiadas en las tres ecorregiones, con el método xtpcse para la Dg posterior al año 2011 (Cuadro 1), y fijando un nuevo

Cuadro 1. Estimación y análisis del modelo para las ecorregiones de Chihuahua

\begin{tabular}{|c|c|c|c|c|c|c|c|c|c|c|}
\hline \multirow{2}{*}{$\begin{array}{l}\text { Coeficientes del } \\
\text { modelo }{ }^{2}\end{array}$} & \multicolumn{10}{|c|}{ Estadísticos/parámetros de postestimación $(P \text {-value })^{1}$} \\
\hline & Regress & & Xtreg & & Xtregar & & Xtgls & & Xtpcse & \\
\hline LnNDVI & 0.7265 & $* \star \star$ & 0.6909 & * & 0.7046 & * & 0.7225 & *** & 0.7141 & *** \\
\hline LnPrecipit & 0.4659 & ** & 0.3010 & * & 0.3192 & * & 0.3428 & ** & 0.3463 & $\star \star \star ~$ \\
\hline LnTemp & -0.3642 & & -0.3868 & & -0.4315 & & -0.6944 & & -0.6933 & * \\
\hline LnSize & -0.8044 & $* \star \star$ & -0.7547 & 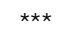 & -0.7444 & $* * *$ & -0.7027 & *** & -0.7020 & *** \\
\hline Ejido & 0.4245 & & 0.9338 & $\star *$ & 0.8809 & $* *$ & 0.5636 & * & 0.5647 & * \\
\hline LnCost & -0.0272 & & -0.0553 & & -0.0543 & & -0.0707 & & -0.0800 & \\
\hline LnProd & 0.1465 & * & 0.1072 & * & 0.1095 & * & 0.1424 & $\star \star \star ~$ & 0.1504 & $\star *$ \\
\hline LnNDVI_Ejido & -0.5417 & $\star \star \star ~$ & -0.3751 & & -0.4018 & & -0.6091 & $\star \star \star$ & -0.5955 & $\star \star \star$ \\
\hline LnCost_ejido & 0.0398 & $\star \star \star ~$ & 0.0302 & & 0.0313 & & 0.0378 & $\star \star \star ~$ & 0.0376 & $\star \star \star *$ \\
\hline LnProd_Ejido & 0.0128 & & -0.0176 & & -0.0145 & & 0.0031 & & 0.0030 & \\
\hline LnNDVI_post2011 & 0.2339 & & 0.1985 & & 0.1999 & & 0.2600 & & 0.2525 & \\
\hline LnProd_post2011 & -0.0368 & & -0.0190 & & -0.0195 & & -0.0270 & & -0.0253 & \\
\hline LnCost_post2011 & -0.0250 & & -0.0267 & * & -0.0269 & * & -0.0275 & ** & -0.0284 & * \\
\hline LnBenef_2010 & 0.0647 & & 0.0595 & ** & 0.0592 & * & 0.0374 & & 0.0359 & * \\
\hline LnBenef_2011 & 0.0215 & & -0.0151 & & -0.0142 & & -0.0224 & & -0.0232 & \\
\hline LnBenef_2012 & -0.0422 & & -0.0311 & & -0.0316 & & -0.0272 & & -0.0254 & \\
\hline post2011 & 1.4655 & $\star \star \star$ & 1.2250 & 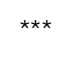 & 1.2359 & $* * \star$ & 1.3812 & *** & 1.3540 & $\star \star \star *$ \\
\hline Zon 2 & -0.4908 & $\star \star \star ~$ & -0.4643 & * & -0.4636 & $* *$ & -0.3240 & $\star \star \star ~$ & -0.3309 & $* * *$ \\
\hline Zon 3 & -0.2842 & * & -0.1702 & & -0.1770 & & 0.0206 & & 0.0112 & \\
\hline Constante & 5.8202 & $\star \star \star ~$ & 6.4112 & $* \star \star$ & 6.4275 & $* * *$ & 6.5840 & $* \star \star$ & 6.5999 & $* \star \star$ \\
\hline $\mathrm{N}$ & 402 & & 402 & & 402 & & 402 & & 402 & \\
\hline $\mathrm{r}^{2}$ & 0.5637 & & 0.5275 & & 0.5331 & & & & 0.7429 & \\
\hline
\end{tabular}

${ }^{1}$ Regress: comando que analiza la variable independiente $x$ observada en el tiempo $t$ para cada unidad de análisis $i$ en un modelo general de regresión lineal; Xtreg: comando que analiza la variable independiente $x$ observada en el tiempo $t$ para cada unidad de análisis $i$ en un modelo regresivo con efecto fijo y aleatorio; Xtregar: comando que analiza la variable independiente $x$ observada en el tiempo $t$ para cada unidad de análisis $i$ en un modelo autoregresivo de grado 1 (AR1) con efectos fijos; Xtgls: comando que analiza la variable independiente $x$ observada en el tiempo $t$ para cada unidad de análisis $i$ en un modelo con estimadores de mínimos cuadrados generalizados factibles; Xtpcse: comando que analiza la variable independiente x observada en el tiempo $t$ para cada unidad de análisis $i$ en un modelo con errores estándar corregidos para panel. Nivel de significancia en los parámetros: ${ }^{*} P<0.05 ;{ }^{* *} P<0.01 ; * * * 00.001$.

${ }^{2}$ La interpretación de los coeficientes o parámetros del modelo están desglosados en el tercer modelo (ecuación 3). 
ciclo de producción y las diferencias en el manejo del hato ganadero (según el tipo de tenencia predial y la zona donde se ubica la explotación), se establecieron los siguientes cinco planteamientos:

1) $\mathrm{Si}$ el NDVI incrementa en una unidad porcentual en las ecorregiones del estado, Dg aumenta 0.7140 unidades porcentuales con un nivel de significancia menor a 0.001 . Un estudio ${ }^{(27)}$ afirma que la disminución del NDVI en zonas semiáridas se traduce en pérdidas no solo económicas, sino ambientales; y una limitante encontrada del NDVI en el monitoreo de la sequía es el desfase entre el déficit de precipitaciones y la respuesta de la variable $^{(28)}$, lo que podría afectar la relación entre la Dg y NDVI.

2) En los predios catalogados como ejidales se identificó una menor capacidad de carga (-0.5955). La variable dependiente (unidades animal/ha/año/predio) posterior al año 2011 no mostró incrementos significativos al aumentar NDVI disponible en las explotaciones; esto debido a la alta precipitación, ya que Dg incrementó 0.3462. Con un nivel de $P<0.05$ los valores de Dg presentaron una disminución de -0.6933 cuando aumentó la temperatura media mensual.

3) Cuando la Dg es más alta en las ecorregiones evaluadas, el porcentaje de unidades de producción con tipo de tenencia ejidal es mayor (0.5646). Para disminuir en ellos el efecto del incremento en los costos netos, se aumentó el número de unidades animal por hectárea (0.0376). Sin embargo, la tendencia posterior al año de sequía (2011) es el decremento de Dg (-0.0283) cuando los costos de producción aumentan una unidad porcentual.

4) Los predios ejidales en condiciones de sequía severa (los cuales no cuentan con capacitación técnica o ingresos estables) revela el aumento del consumo de materia vegetal para conservar el número de cabezas en el hato, sin importar el sacrificio de los pastizales ${ }^{(29)}$. El número de capacitaciones técnicas mostró un efecto significativo $(P<0.05)$ sobre el comportamiento de la Dg en las ecorregiones. Lo anterior posiblemente por efecto de ajuste en la carga animal. Sin embargo, para el año 2010 existió un incremento de 0.0358 en las unidades animal por hectárea en Chihuahua; esto puede ser por efecto del repoblamiento del hato $^{(25)}$.

5) La zona semiárida de Chihuahua indicó una pérdida en $\mathrm{Dg}$ en comparación con la zona desértica (-0.3305; $P<0.001)$. Con relación al tamaño de las explotaciones, se puso en evidencia que los predios con más terreno manejaron una menor carga ganadera (-0.7019).

Existió una recuperación de las unidades animal por hectárea posterior al año de sequía y, se obtuvo un incremento del 1.3535; sin embargo, este aumento se reflejó hasta el año 2013. En el 2012 existieron pérdidas marcadas, principalmente en los primeros meses del año. Este método sólo considera la disposición espacial de los individuos para hacer los conglomerados ${ }^{(30)}$. Por lo anterior, para el año 2012 los egresos se incrementaron, posiblemente por gastos de suplementación energética y vitamínica del ganado. La producción, entendida de ganado, refleja un decremento notorio (Figura 1).

La recuperación para el año 2013 hace suponer que existió un nuevo ciclo de recuperación en los pastizales y una inversión asociada, reflejada en los egresos registrados con respecto a la producción económica de los predios ganaderos. Así mismo, se informa $^{(31)}$ que parámetros sobre los coeficientes técnicos relacionados con las necesidades de alimentación, son relativos a los aportes nutricionales y el crecimiento del pasto.

En este sentido, diversos estudios $(32,33,34)$ demuestran que el aumento en el riesgo sobre los patrones de recuperación de pastizales es influenciado por factores como cambios en uso del suelo, tecnificación y capacitación de productores ${ }^{(34)}$; así mismo, los cambios climáticos extremos (sequía y temperaturas extremas) afectan la productividad de los pastos ${ }^{(35,36)}$, que directamente impactan la productividad ganadera en las ecorregiones.

\section{CONCLUSIONES E IMPLICACIONES}

Se generó un modelo (ecuación 3) con indicadores socioeconómicos y ambientales para el comportamiento de la densidad ganadera en las ecorregiones del estado de Chihuahua; existió un 
Figura 1. Tendencias de la producción y egresos ganaderos en el estado de Chihuahua respecto a la densidad ganadera

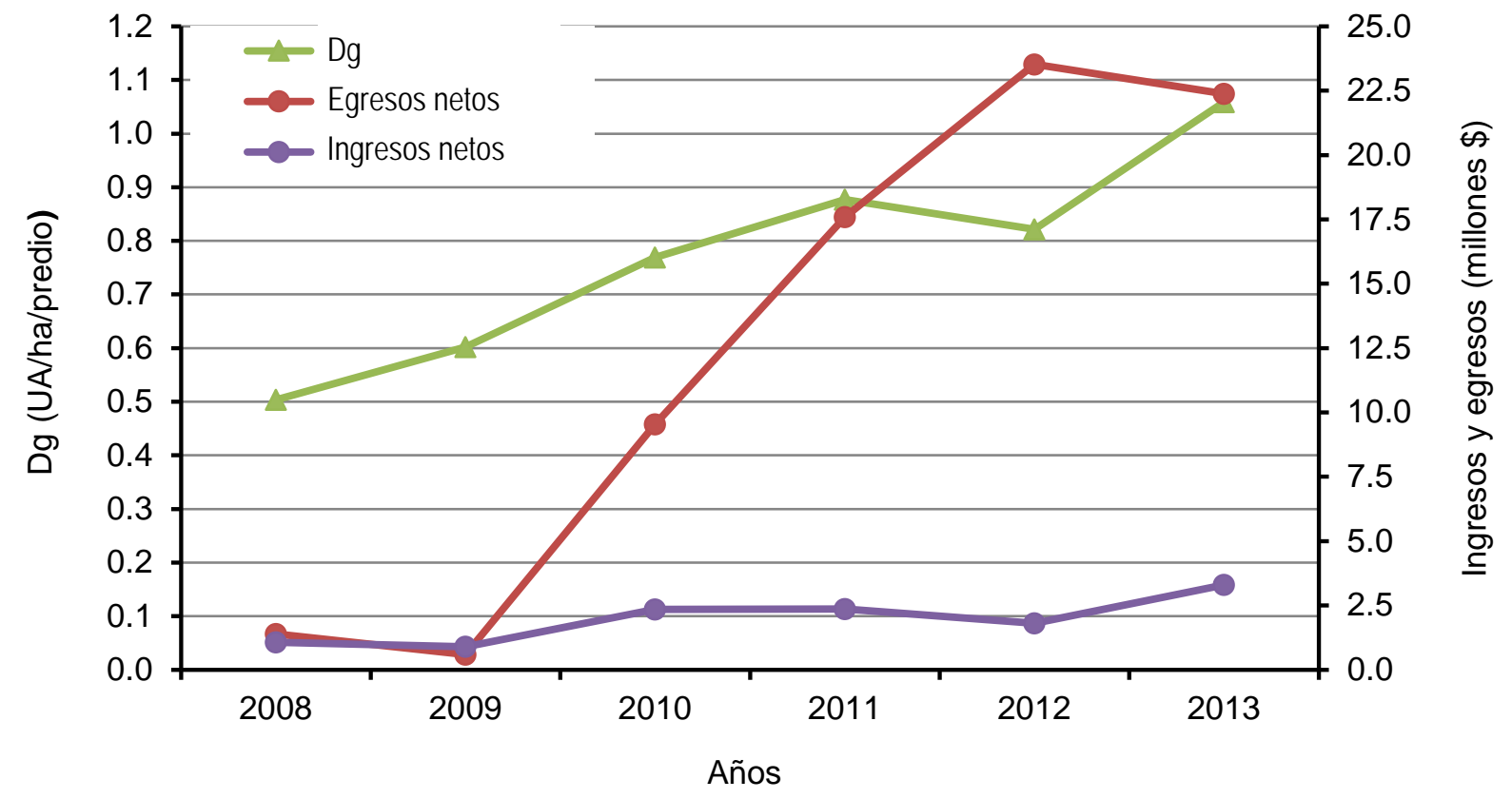

$\mathrm{Dg}=$ densidad ganadera; $\mathrm{UA}=$ unidad animal (miles).

cambio estructural en la densidad ganadera, la disponibilidad vegetal y la capacidad de carga animal por hectárea disminuyeron. La estimación de la densidad indicó una recuperación posterior al periodo de sequía, y se esperaría que la disponibilidad de los pastos mejore con la recuperación vegetal en un nuevo ciclo ambiental (manteniendo el resto de variables constantes). Los fenómenos meteorológicos extremos presentaron un cambio estructural en 2012; además, la vulnerabilidad no siempre está relacionada con el tipo de tenencia predial, sino que las variables de capacitación rural a productores y el ingreso per cápita, influyen en la densidad ganadera. Las nuevas tecnologías geoestadísticas y modelos econométricos facilitan el modelado para la gestión de riesgos e impacto de la sequía en la ganadería extensiva.

\section{AGRADECIMIENTOS}

Se agradece al Consejo Nacional de Ciencia y Tecnología, al Centro de Extensión e Innovación Rural Noroeste y al CEIGRAM en la Universidad Politécnica de Madrid donde se realizó una estancia durante los meses de junio a diciembre del año 2014 en los cuales se dispuso de apoyo en infraestructura, equipo y asesoramiento académico para desarrollar gran parte el análisis de esta investigación.

\section{LITERATURA CITADA}

1. Valerio VA. Distribución, extensión espacial y condición de los pastizales en el Estado de Chihuahua [tesis maestría]. Chihuahua, México: Universidad Autónoma de Chihuahua; 2005.

2. Royo $M$, Melgoza $A$, Santos J, Carrillo R, Jurado $P$, Gutiérrez $P$, Echavarria F. La salud de los pastizales medianos en los estados de Chihuahua y Zacatecas [resumen]. Simposio Internacional de Manejo de Pastizales. Zacatecas, Zacatecas. 2011:78.

3. Aguirre CJ. Estado actual de los pastizales del desierto chihuahuense y estrategias para su conservación y uso sustentable [tesis doctoral]. Chihuahua, México: Universidad Autónoma de Chihuahua; 2012.

4. Díaz-Solís H, Kothmann MM, Hamilton WT, Grant WE. A simple ecological sustainability simulator (SESS) for stocking rate management on semi-arid grazinglands. Agr Syst 2003; 76(2):655680.

5. Pinedo AC, Hernández QNS, Melgoza CA, Rentería VM, Vélez SVC, Morales NC, et al. Diagnóstico actual y sustentabilidad de los pastizales del estado de Chihuahua ante el cambio climático [boletín informativo]. Chihuahua, México: Universidad Autónoma de Chihuahua; 2013.

6. Bielza M, Conte C, Dittmann C, Gallego J, Stroblmair J, Catenari $\mathrm{R}$, Dittmann C. Risk manangement and agricultural insurance schemes in Europe. [boletín informativo]. Brucelas, Bélgica; 2009. 
Ireyli Zuluamy I racheta-Lara, et al. / Rev Mex Cienc Pecu 2017;8(2):193-200

7. Mary K. Water use and water management policy in the Chihuahua Desert Ecoregion [boletín informativo]. Texas Center for Policy Studies. El Paso, Texas; 2002.

8. CONAGUA. Comisión Nacional del Agua. Reporte anual 2012. Servicio Meteorológico Nacional. Gerencia Meteorología y Climatología Subgerencia de Pronóstico a Mediano y Largo Plazo. Chihuahua, México. 2012.

9. NADM. Monitor de Sequía de América del Norte. Informe mensual a gran escala de la sequía en América del Norte. http://www.ncdc.noaa.gov/nadm.html. Consultado 20 Ene, 2014.

10. PACP-Ch. Plan de acción para la conservación y uso sustentable de los pastizales del desierto chihuahuense en el Estado de Chihuahua 2011-2016. Guzmán-Aranda JC, et al, editores. Comisión para la Cooperación Ambiental de América del Norte. Gobierno del Estado de Chihuahua. 2011.

11. CRED. Center for Reasearch on the epidemiology of disaster (Base de datos de desastres internacionales). http://www.emdat.be/ database. Consultado 10 Oct, 2014.

12. Financiera-Rural. Bovino y sus derivados. Dirección General Adjunta de Planeación Estratégica y Análisis Sectorial. http://www. financierarural.gob.mx/Paginas/Financieraruralini.aspx. Consultado 5 Ago, 2013.

13. SAGARPA. Secretaría de Agricultura, Ganadería, Desarrollo Rural, Pesca y Alimentación. Manejo sustentable de los pastizales. http://www.sagarpa.gob.mx. Consultado 4 Ene, 2014

14. SIAP. Servicio de Información agroalimentaria y Pesquera. Producción pecuaria y agrícola para el año 2012. http://www.siap.gob.mx/. Consultado 27 Oct, 2014.

15. Eastman JR. IDRISI Kilimanjaro Guide to GIS and image processing. Clark University. https://www.mtholyoke.edu/courses /tmillett/course/geog307/files/Kilimanjaro\%20Manual.pdf. Accessed Sep 20, 2014.

16. Hennessy K, Fawcett R, Kirono D, Mpelasoka F, J ones D, Bathols J, et al. An assessment of the impact of climate change on the nature and frequency of exceptional climatic events. Drought exceptional circumstances. Australian Government Bureau of Meteorology [bulletin informative]. Canberra, Australia: Australian Government; 2008.

17. Sastre HJ, Rodero E, Rodero A, Herrera M, Peña F. Caracterización etnológica y propuesta del estándar para la raza bovina colombiana Criolla Casanare. Animal Genetic Resources. Food and Agriculture Organization of the United Nations. Roma, Italia. 2010;77(3):7379.

18. Chuvieco E. Teledetección ambiental. 3ra ed. Barcelona, España: Editorial Ariel SA; 2010.

19. Villa-Herrera A, Paz-Pellat F, Pérez-Hernández MJ, Rojas-Montes C, Rodríguez AM, Ortiz-Acosta S, et al. Estimación de la capacidad de carga animal en agostaderos usando un índice de vegetación de pendientes normalizadas. Agrociencia 2014;48(6):599-614.
20. SIMA-COAHUILA. Monitoreo de agostaderos y pastizales https://sites.google.com/a/sima-coahuila.com/agostaderos-decoahuila/. Consultado 28 Ago, 2014.

21. INEGI. Instituto Nacional de Estadística, Geografía e Informática. Manejo sustentable de pastizales. http://www.patrocipes.org.mx/ publicaciones/pastizales/SimposioPastizales6/pastizala.pdf. Consultado 30 Ago, 2014

22. RAN. Registro Agrario Nacional. Registro público de la propiedad 2013. http://www.ran.gob.mx. Consultado 25 Abr, 2014.

23. SAGARPA. Secretaría de Agricultura, Ganadería, Desarrollo Rural, Pesca y Alimentación. Alternativas para el manejo de la sequía en ranchos ganaderos del Norte de México. http://www. sagarpa.gob.mx/Paginas/default.aspx. Consultado 20 Ago, 2014.

24. SAGARPA. Secretaría de Agricultura, Ganadería, Desarrollo Rural, Pesca y Alimentación. Informe de rendición de cuentas de la administración pública federal 2006-2012. http://inicio.ifai. org.mx/Rendicion_Cuentas/Primera\%20etapa\%202006_2012.pdf. Consultado 24 Sep, 2014.

25. STATACorp. Stata Statistical Software: Release 12. College Station, TX: StataCorp LP. 2011.

26. ESRI. ArcGIS Desktop: Release 9.3. Redlands, CA: Environmental Systems Research Institute. 2011.

27. Ares J. Systems valuing of natural capital and investment in extensive pastoral systems: lessons from the Patagonia case. Ecol Econ 2007; 52(3): 162-173.

28. Tucker CJ, Sellers PJ. Satellite remote sensing of primary production. J Remote Sens 1986; 11:1395-1416.

29. Kazianga $\mathrm{H}$, Udry $\mathrm{C}$. Consumption smoothing? Livestock, insurance and drought in rural Bukina Fao. J Dev Econ 2006; 79:413-446.

30. Kriegel H, Kröger P, Sander J, Zymek A. Density-based clusteringWRIEs Data Mining Knowledge Discovery. 2011;13(3):231-240.

31. Martín-Polo JL, Gutiérrez Cl, Blanco de Pablos A, SánchezRodríguez ME. La dehesa y los recursos forrajeros: fertilización laboreo y siembra. J Agric Res 2003; 1(1):35-42.

32. Halcrow $\mathrm{H}$. Actuarial structures for crop insurance. J Forn Econ 1949; 31():418-448.

33. Dracup J, Lee K, Paulson JE. On the statistical characteristics of the droughts events. Weater Res 1980;16(3):289-296.

34. Chantarat S, Barret CB, J anvilisric T, Mudsrid S. Index insurance for pro-poor conservation of hornbills in Thailand. Proc Int Congr Ecol 2011;108(34):51-56.

35. IPCC. Grupo Intergubernamental de Expertos sobre el Cambio Climático. Cambio climático 2007: Informe de síntesis. 1ra ed. Ginebra, Suiza; 2007.

36. Holman IP. Climate change impacts on groundwater rechargeuncertainty, shortcomings, and the way forward?. Hydrogeol J 2006; 14(5):637-647. 